\title{
Study of Antidepressant Activity of Garcinia indica(Kokum) Fruit Rind in Wistar Albino Rats
}

\author{
Bhagyashree Ajjakana ${ }^{1}$, Roopa Prasad Nayak ${ }^{2}$ \\ 1,2 Department of Pharmacology, Yenepoya Medical College, \\ Yenepoya (Deemed to Be University), Mangaluru, Karnataka, India.
}

\section{ABSTRACT}

\section{BACKGROUND}

Depression is a mental disorder which is treatable but detected less often in primary healthcare settings. Therefore, there is a need for an effective treatment strategy for the management of depression. Garcinia indica (Thouars) Choisy is a slender evergreen tree. An invitro animal study has shown that its phytochemical constituent, hydroxy citric acid has the ability to increase serotonin levels in the brain. Hence, the objective of the study was to evaluate the antidepressant activity of ethanolic extract of Garcinia indica fruit rind in animal models of depression and compare it with control and standard drugs, imipramine, and fluoxetine.

\section{METHODS}

The study was conducted on Wistar albino rats of either sex. The animals were grouped into five, containing six animals in each group. Control $(0.1 \%$ carboxymethylcellulose, $10 \mathrm{ml} / \mathrm{kg}$ ), ethanolic extract of Garcinia indica (GIEE 1 ) $250 \mathrm{mg} / \mathrm{kg}$, ethanolic extract of Garcinia indica $\left(\mathrm{GIEE}_{2}\right)-500 \mathrm{mg} / \mathrm{kg}$, Standard ${ }_{1}$ Imipramine - 10mg/kg ( Forced Swim test only) and Standard 2 - Fluoxetine $20 \mathrm{mg} / \mathrm{kg}$ (Tail suspension test only). Drugs were administered for 14 days and antidepressant activity was evaluated on the $14^{\text {th }}$ day after one hour of drug administration using two models - Forced swim test and tail suspension test. Results were tabulated as mean \pm SEM (standard error of mean). One-way analysis of variance (ANOVA) followed by Tukey Kramer test was used to interpret the statistical significance.

\section{RESULTS}

The period of immobility was obtained as $21.83 \pm 1.44$ and14.66 \pm 2.74 in forced swim test and $36.8 \pm 1.01$ and $14.3 \pm 0.954$ in tail suspension test in GIEE 1 and $\mathrm{GIEE}_{2}$ treated groups respectively, which was significantly less compared to control.

\section{CONCLUSIONS}

Garcinia indica has significant antidepressant activity compared to the control.
Corresponding Author: Dr. Roopa Prasad Nayak, Professor and HOD, Department of Pharmacology, Yenepoya Medical College, Mangaluru, Karnataka, India. E-mail: roopapnayak@yenepoya.edu.in

DOI: $10.14260 / j e m d s / 2021 / 628$

How to Cite This Article:

Ajjakana B, Nayak RP. Study of antidepressant activity of Garcinia indica(Kokum) fruit rind in Wistar albino rats. J Evolution Med Dent Sci 2021;10(36): 3077-3082, DOI:

10.14260/jemds/2021/628

Submission 11-06-2021, Peer Review 05-08-2021, Acceptance 11-08-2021, Published 06-09-2021.

Copyright (C) 2021 Bhagyashree Ajjakana et al. This is an open access article distributed under Creative Commons Attribution License [Attribution 4.0 International (CC BY 4.0)]

\section{KEY WORDS}

Antidepressant, Garcinia indica, Fruit Rind, Wistar Albino Rats 


\section{BACKGROUND}

Depression is a mental disorder which is treatable but detected less often in primary health care settings. It is estimated that about $10-15 \%$ of people suffer from major depression worldwide. The prevalence of depression in India has shown figures similar to western countries that is, about $15.9 \%{ }^{1}$

Treatment gap between various disorders affecting mental health is between 70 and $90 \% .^{2}$ Therefore, there is a need for an effective treatment strategy for the management of mental illness especially depression. Even though the presently available antidepressant medications help to improve the overall quality of life of the people, they are associated with many untoward effects which are a drawback and lead to their discontinuation.

Some of the side effects are nausea, diarrhoea, agitation, insomnia, sweating, dryness of mouth and blurred vision. ${ }^{3}$ About $75-80 \%$ of the population in the world depend mainly on herbal drugs for their primary healthcare. This is mainly due to the belief that herbal drugs are locally available and cheap and without any untoward effects. The present modern medicine has its basis on the utilization of plant medicine.

Many commonly used drugs have been prepared from plant sources, examples being aspirin (from willow bark), morphine (from opium poppy), digoxin (from foxglove) and quinine (from cinchona bark).

World Health Organization (WHO) has expressed that the utilization of herbal remedies all over the world surpasses the conventional drugs by two to three times. ${ }^{4}$

Some of the herbal drugs are already well known for the treatment of psychiatric illness over the past decade. St John's wort and kava have proved their effectiveness in the treatment of various psychiatric illnesses. ${ }^{5}$ Because of the expanding popularity of herbal drugs in the present scenario, many patients prefer consulting herbalists and naturopaths along with physicians.

Still further studies are required to understand their efficacy in various mental illnesses. These herbal drugs can serve as an alternative therapy to conventional non herbal drugs in people who do not tolerate them as herbal drugs are considered safer. ${ }^{6}$

Garcinia is a very large genus containing numerous trees and shrubs possessing many medicinal properties. More than 200 species belong to this genus, out of which 30 different trees and shrubs are said to be grown in India. Garcinia indica(Thouars, Choisy) (common name - kokum) is a slender everlasting tree which is native to our country. ${ }^{7}$ Garcinia indica tree is most commonly grown in the humid region of the Western Ghats region of South India. It is widely distributed along the Konkan region including Goa, Dakshina Kannada and Coorg district in Karnataka, North Malabar in Kerala.

It is also found in certain areas of West Bengal and Assam. It is usually cultivated in a limited number along with other plants and trees. On the whole, the tree can be grown in any kind of soil which can withstand moisture for long duration. $8,9,10,11$

Each part of the tree has got medicinal properties. ${ }^{12}$ It is also considered as an Indian superfood.
Garcinia indica is used as a home remedy for treating various conditions like diarrhoea, dysentery, haemorrhoids, asthma, gastritis, colicky pain abdomen, indigestion problems, flatulence, dermatitis, ulcer and helminthic infestations. ${ }^{8,12,13}$

It is also considered as a functional food because it not only provides additional nutrition but also health benefits to the consumers. The nutritional profile of Garcinia indica is responsible for its antioxidant activity. There is an increasing demand for the functional food due to current nutrition trend in our country.

Garcinia indica can serve as a promising functional food because of its nutritional content.9,13 The major phytochemical constituents in Garcinia indica are hydroxy citric acid, garcinol and anthocyanin pigment. All these are present mainly in the rind of the kokum fruit. These constituents possess numerous medicinal properties.8,9,10,13

An invitro animal study has shown that hydroxy citric acid has the ability to increase serotonin levels in the brain. $7,14,15$ Hence, this study has been conducted to evaluate the antidepressant activity of ethanolic extract of Garcinia indica fruit rind in preclinical models.

\section{Objective}

The objective of the study is to evaluate the effect of ethanolic extract of Garcinia indica fruit rind on animal models of depression and compare it with control and standard drugs, imipramine, and fluoxetine.

\section{METHODS}

This is a pre-clinical study carried out in Wistar albino rats. The study was initiated after obtaining the ethics clearance from the Institutional Animal Ethics Committee. The study was conducted in the Ethnopharmacology lab, Department of Pharmacology, Yenepoya Medical College, Mangaluru from February 2015 to April 2015.

\section{Plant Material}

Fruit rinds of Garcinia indica were used for the study. Fruits were collected in the month of March and April from the Garcinia indica tree grown in Dakshina Kannada district. Botanical authentication was done by a botanist. Fruits were washed and fruit rinds were separated from the pulp and were shade and air dried for a period of 3 weeks. A mixer grinder was used to convert the dried fruit rind into powder form.

\section{Preparation of the Extract}

The powder was taken for extraction in Soxhlet apparatus using $95 \%$ ethanol $^{16}$ as a solvent. Temperature was maintained around $60-70^{\circ} \mathrm{C}$. The extract was concentrated in the rotavapor. The resultant brown coloured extract weighed $184.8 \mathrm{~g}$. The yield was $46.2 \% \mathrm{w} / \mathrm{w} .0 .1 \%$ sodium salt of carboxymethyl cellulose in distilled water ${ }^{17}$ was used as solvent to dissolve the extract and was administered to animals in various doses. 


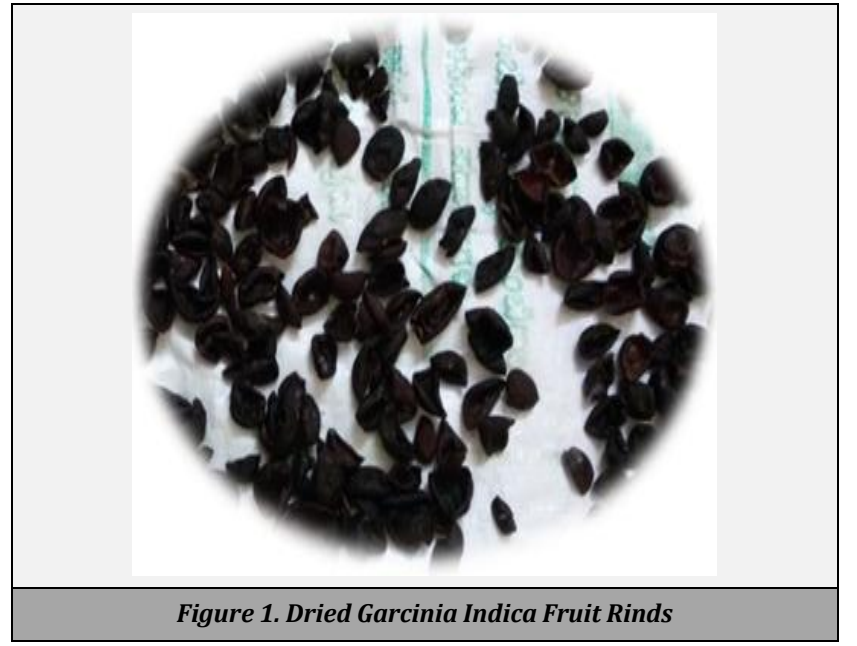

\section{Dose of the Extract}

Two doses were taken for conducting the antidepressant study $-250 \mathrm{mg} / \mathrm{kg}$ and $500 \mathrm{mg} / \mathrm{kg} .{ }^{7}$ Quantity of the test drug/extract administered was calculated according to the body weight of the animals and was administered orally for a period of 14 days. On the $14^{\text {th }}$ day, after one hour of drug administration, animals were tested for antidepressant activity.

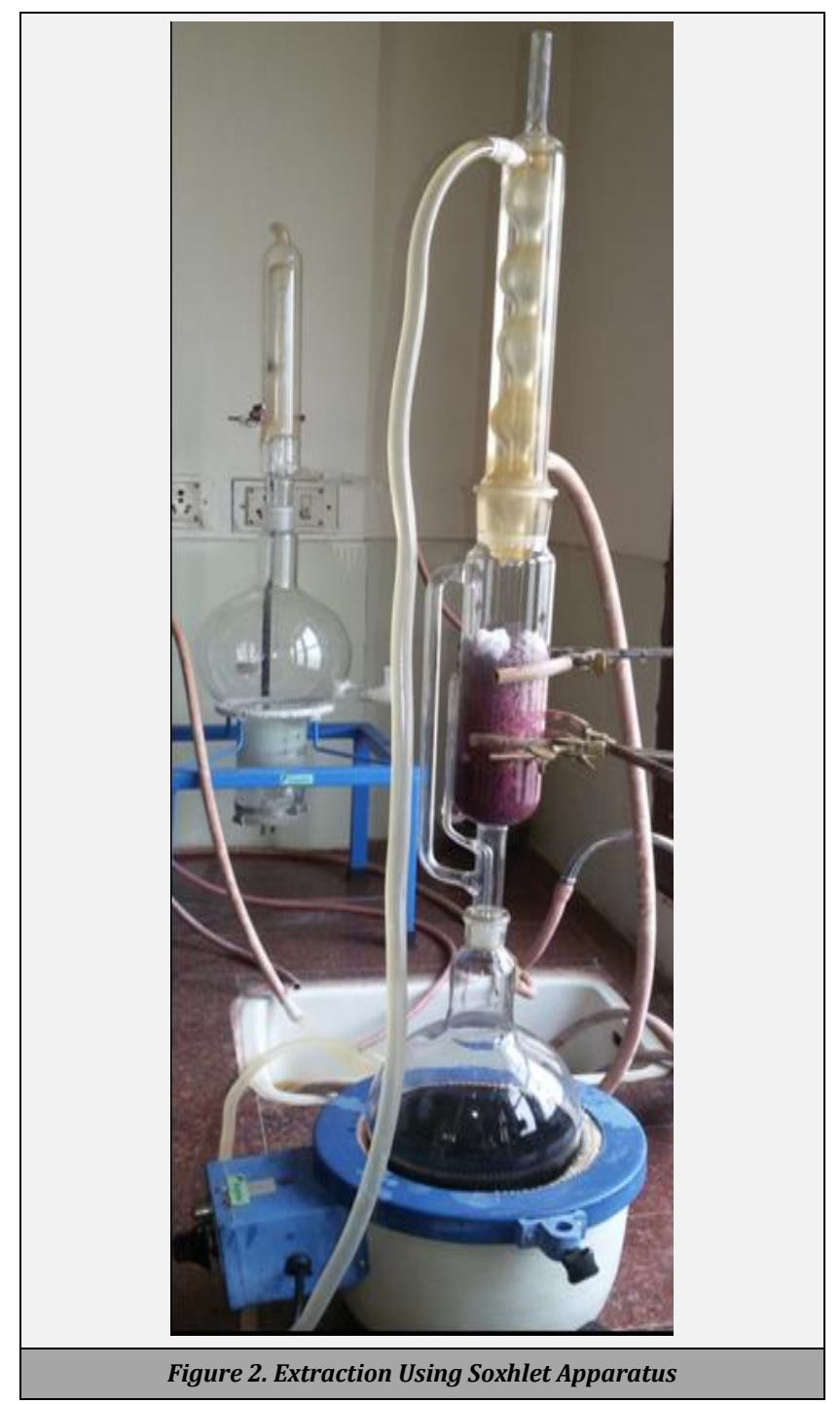

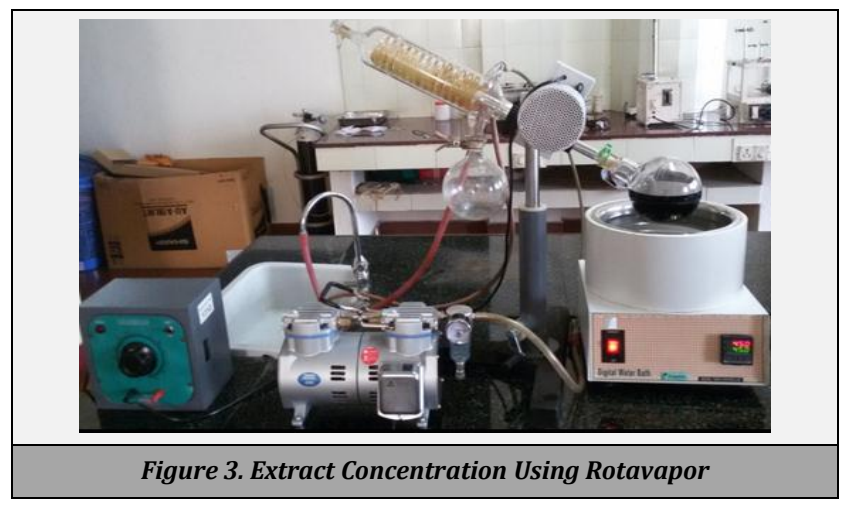
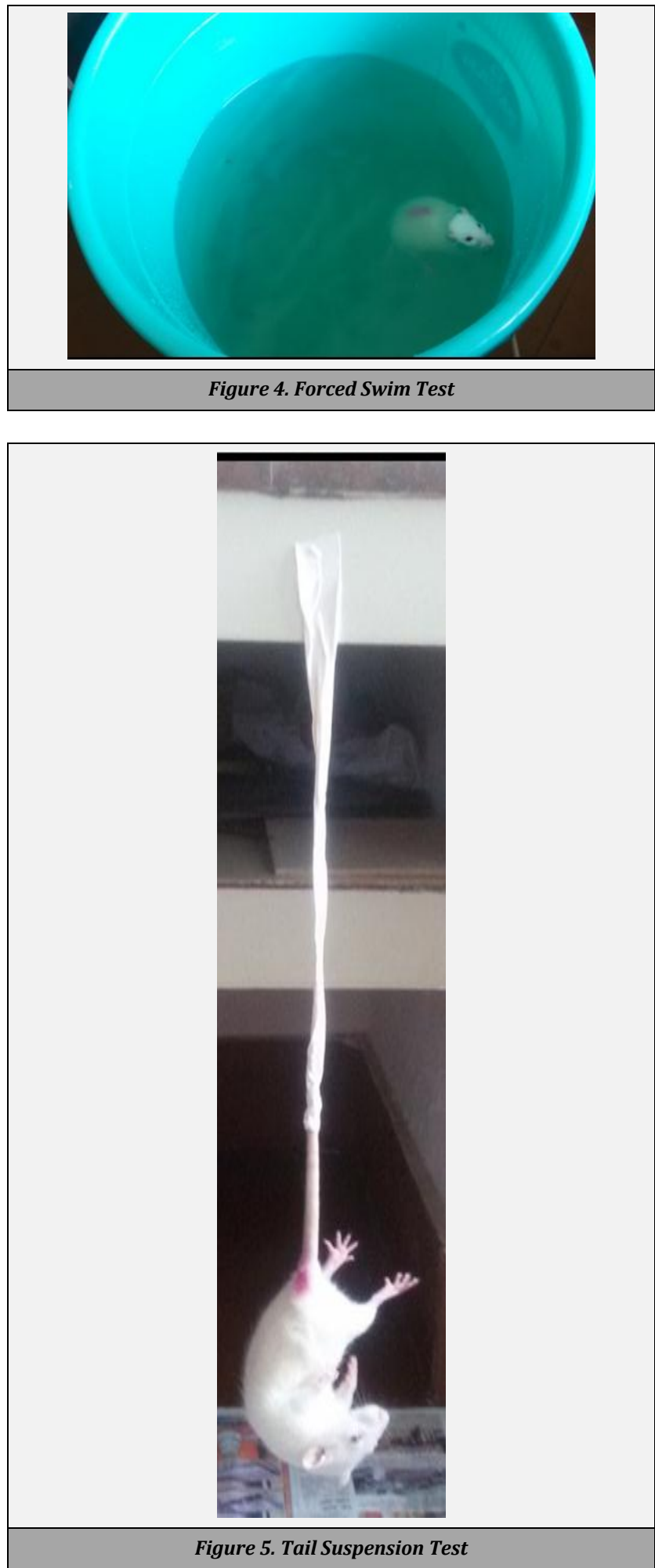


\section{Sample Size and Study Design}

Wistar albino rats of either sex, aged about 3 - 4 months and weighing around 150 - 200gms were used in this study. Animals were housed under standard conditions in the animal house with temperature maintained around $24+/-2^{\circ} \mathrm{C}$ with 12: 12 hour light: dark cycle. Standard pellet food and water were provided to the animals. The guidelines laid by CPCSEA (Committee for the Purpose of Control and Supervision of Experiments on Animals) were followed while handling the animals. The animals were grouped into five, containing six animals in each group. Sample size of minimum six animals in each group is necessary to achieve statistically significant results.
- Group I

carboxymethylcellulose $(10 \mathrm{ml} / \mathrm{kg})^{17}$

- Group II - Ethanolic extract of Garcinia indica(GIEE 1 ) $250 \mathrm{mg} / \mathrm{kg}^{7}$

- Group III - Ethanolic extract of Garcinia indica(GIEE2) $500 \mathrm{mg} / \mathrm{kg}^{7}$

- Group IV - Standard 1 - Imipramine - 10mg/kg18 (Standard drug in Forced Swim test only)

- Group V - Standard 2 - Fluoxetine - 20mg/kg19 (Standard drug in Tail suspension test only)

Drugs were administered orally for 14 days and antidepressant activity was evaluated on the $14^{\text {th }}$ day after one hour of drug administration.

\section{Forced Swim Test ${ }^{18}$}

Each rat was brought into the container filled with water. They were constrained to swim in the water and were observed for 6 minutes. Rat was considered immobile when it was floating in the water freely or making only least movements to keep its head over the water. Duration of immobility was noted for each rat. Antidepressant activity was described by increase in the duration of mobility or decrease in the duration of immobility.

\section{Tail Suspension Test ${ }^{19}$}

Each rat was suspended from the edge of a slab by an adhesive tape placed approximately $1 \mathrm{~cm}$ from the tip of the tail. They were observed for 6 minutes. Rat was considered immobile when it was hanging freely without making any movements. Duration of immobility was noted for each rat. Antidepressant activity was described by increase in the duration of mobility or decrease in the duration of immobility.

\section{Statistical Analysis}

Statistical software, GraphPad InStat was used to analyse the data. Results were tabulated as mean \pm SEM (standard error of mean). One-way ANOVA followed by Tukey Kramertest was used to interpret the statistical significance. $\mathrm{P}$ value< 0.01 was considered as highly significant and $P$ value $<0.001$ was considered as very highly significant.

\section{RESULTS}

\section{Forced Swim Test}

GIEE (Garcinia indica ethanolic extract) showed dose dependent decrease in the period of immobility when compared to the control group. Period of immobility were not significantly different from the standard group. GIEE at the dose of $250 \mathrm{mg} / \mathrm{kg}$ showed decrease in period of immobility compared to control which was highly significant. GIEE at the dose of $500 \mathrm{mg} / \mathrm{kg}$ showed decrease in period of immobility compared to control which was very highly significant.

\begin{tabular}{|ccc|}
\hline Sl. No. & Group & Period of Immobility (Seconds) \\
1 & Control $^{-}$ & $76.5 \pm 18.46$ \\
2 & GIEE $_{1}$ & $21.83 \pm 1.44^{\mathrm{a}}$ \\
3 & GIEE $_{2}$ & $14.66 \pm 2.74^{\mathrm{b}}$ \\
4 & Imipramine & $13.83 \pm 2.79$ \\
\hline \multicolumn{3}{|c|}{ Table 1. Forced Swim Test } \\
\hline GIEE $_{1}$ - Ethanolic extract of Garcinia indica $250 \mathrm{mg} / \mathrm{kg}$ \\
GIEE - Ethanolic extract of Garcinia indica $500 \mathrm{mg} / \mathrm{kg}$ \\
One-way ANOVA followed by Tukey Kramer test \\
ap value $<0.01$ - highly significant, compared to I \\
bP value $<0.001$ - very highly significant, compared to I \\
\hline
\end{tabular}

\section{Tail Suspension Test}

GIEE (Garcinia indica ethanolic extract) showed dose dependent decrease in the period of immobility when compared to the control group. There was a significant difference in the periods of immobility observed between the two doses of GIEE. GIEE at the dose of $250 \mathrm{mg} / \mathrm{kg}$ and $500 \mathrm{mg} / \mathrm{kg}$ showed decrease in period of immobility compared to control which was very highly significant. GIEE at the dose of $500 \mathrm{mg} / \mathrm{kg}$ showed decrease in period of immobility which was very highly significant compared to GIEE at $250 \mathrm{mg} / \mathrm{kg}$.

\begin{tabular}{|ccc|}
\hline SI. No. & Group & Period of Immobility (Seconds) \\
\hline 1 & Control & $73.8 \pm 2.88$ \\
2 & GIEE $_{1}$ & $36.8 \pm 1.01^{\mathrm{a}}$ \\
3 & GIEE $_{2}$ & $14.3 \pm 0.954^{\mathrm{a}, \mathrm{b}}$ \\
4 & Fluoxetine & $10 \pm 1.80$ \\
\hline \multicolumn{3}{|c|}{ Table 2. Tail Suspension Test } \\
\hline GIEE $_{1}$ - Ethanolic extract of Garcinia indica 250mg/kg \\
GIEE $_{2}$ - Ethanolic extract of Garcinia indica 500mg/kg \\
One-way ANOVA followed by Tukey Kramer test \\
aP value< 0.001 - very highly significant, compared to I \\
bP value< 0.001 - very highly significant, compared to II \\
\hline
\end{tabular}

\section{DISCUSSION}

Depression is characterized by low mood, loss of appetite, irritability, and difficulty in focusing. Depression is a chronic psychiatric condition. Many of the depressed patients present with suicidal ideation. Depressed patients are at the risk of developing various chronic medical disorders like coronary artery diseases and type II diabetes mellitus. ${ }^{20}$ Clinical manifestation of depression varies between individuals in a variety of ways. In younger individuals, it might manifest as loss of self-interest and to the surroundings. In older individuals, the presentation might be decreased in mental functioning which might mimic dementia. In some other cases, there might be no symptoms at all but patient might have committed suicide. Other features which are considered to be core symptoms of depression are loss of sleep and appetite, loss of focus and change in the smoothness of motor 
activity. Depression can be categorized into two - reactive depression and endogenous depression. Reactive depression is caused due certain stressful events, whereas endogenous depression is not associated with any precipitating events. In endogenous depression, there might be brain pathology and specific intervention is necessary. In reactive depression, counseling might be sufficient. Regardless of the varieties, both respond to antidepressant agents. As there are certain diagnostic features of depression, it is comparatively easier to diagnose depression than other psychiatric disorders. Treatment of depression is a must as suicidal tendency might be a devastating endpoint in some cases. ${ }^{21}$ In spite of the wide prevalence of depression, the information regarding the pathophysiological and neurobiological factors contributing to it is still under infancy. Depression by itself is a chronic condition like any other medical disorder of longer duration. ${ }^{20}$ There are various hypotheses explaining the pathophysiology of depression. Among them, monoamine hypothesis is the most common. ${ }^{22}$ This is because, majority of the antidepressant drugs used in clinical practice indirectly increase the monoamine levels in the synapse by either inhibiting their re-uptake or by inhibiting their degradation. Serotonin, noradrenaline and dopamine are the significant monoamine neurotransmitters involved in depression. ${ }^{20,22}$ The hypothesis stating the association between serotonin and depression is almost half a century old. It is undoubtedly known that serotonin depletion plays an important role in the pathophysiology of depression. This is again substantiated by the fact that selective serotonin reuptake inhibitors (SSRIs) which increase the serotonin level, plays a role in the treatment of depression. ${ }^{23}$ Serotonin levels are found to be lower in schizophrenic patients with suicidal tendencies compared to schizophrenic patients with nonsuicidal tendency and also serotonin metabolites in brain and spinal fluid were found to be higher in depressed patients with suicidal tendency. In this context, it can be inferred that reduction of serotonin might be associated with suicidal ideation rather than depression itself. Even though serotonin may not be directly associated with depression, there are some evidences to suggest that serotonin derangement contributes to the development of depression and increase in serotonin levels contribute in some way to the process of recovery of depression at the molecular and cellular levels. ${ }^{21}$ Although drugs increase the monoamine levels and improve depressive symptoms, they also cause numerous adverse effects like dry mouth, weight gain, sweating etc. and also the clinical response is not attained immediately. ${ }^{24}$ Therefore, there is a need of an antidepressant drug with better clinical effect and less adverse effects.

Herbal medicine possesses prime importance in primary healthcare in our country. Plant materials can be a potential source to manufacture novel compounds. But the beneficial effect has to be appropriately tested for its efficacy and safety before concluding their superiority over non herbal drugs. Without appropriate preclinical and clinical studies, one cannot recommend the use of traditional medicine. Various parts of Garcinia indica are used as a home remedy for treating various conditions like diarrhoea, dysentery, haemorrhoids, asthma, gastritis, colicky pain abdomen, indigestion problems, flatulence, dermatitis, ulcer and helminthic infestations. It is also being used as an anti-obesity agent, anti-inflammatory and analgesic agent, cardiotonic, hepato-tonic, antitumor, anti-perspirant, astringent, demulcent and emollient since ages. ${ }^{8,12,13}$

Various animal models are present for demonstrating antidepressant activity of potential drugs among which forced swim test and tail suspension test were the two animal models used to evaluate the antidepressant activity of GIEE. In both the models, period of immobility was the parameter used to assess the antidepressant activity. Decrease in the period of immobility was considered as an indicator of antidepressant activity.

Forced swim test was described by Porsolt. Forced swim test is at present considered as one of the reliable models for testing potential antidepressant drugs. Rodents are forced to swim in a restricted compartment of height $40 \mathrm{~cm}$, diameter $18 \mathrm{~cm}$ with $15 \mathrm{~cm}$ of water at $25^{\circ} \mathrm{C}$ temperature from which escaping is impossible. After initial few minutes of struggling, they assume a floating posture without any swimming movements or just minimal movements to keep their head above the water level. This state is characterized by depressive behaviour which can be reduced by potential antidepressant drugs. ${ }^{25}$ This test also has the advantage that it is a reasonable model for differentiating antidepressants from neuroleptics and anti-anxiety drugs. Also, it provides a platform for assessing various neurobiological and genetic mechanisms influencing stress and antidepressant responses. Forced swim test is highly sensitive to monoaminergic transmission. ${ }^{26}$ Various literatures suggest drugs that influence serotonin transmission increase the swimming behaviour in rats. ${ }^{27}$ GIEE showed dose dependent decrease in the period of immobility compared to the control which was statistically significant, suggesting antidepressant action.

Tail suspension test is another important model for evaluating activity of various potential antidepressant agents. Rodents are made to hang from the edge of a table at a height of $58 \mathrm{~cm}$ from the floor, adhesive tape being placed approximately $1 \mathrm{~cm}$ from the tip of the tail and observed for initiation of immobility after few minutes of struggling. This immobility is characterized by state of despair which can be reduced by potential antidepressant agents. ${ }^{25}$ This test is considered as a 'dry version' of forced swim test because the parameter assessed in tail suspension test is identical to forced swim test although the procedure done is exclusively different. This test is also specific to antidepressants as it differentiates antidepressants from other drugs used for psychosis, anxiety and attention deficit hyperactivity disorder. Previous studies indicate a genetic background in the behavioural responses observed in tail suspension test. ${ }^{28}$ GIEE showed significant decrease in the period of immobility compared to control. The decrease in period of immobility was dose dependent i.e., higher dose showed maximum decrease in the period of immobility.

The phytochemical constituent, garcinol, possesses antioxidant potential preventing free radical formation and cell injury. Another phytochemical constituent, anthocyanin, is the major pigment which provides purple colour to the rind of kokum fruit. Anthocyanins are also known to possess free radical scavenging property which proves its antioxidant potential.9,10 Hydroxy citric acid is also known as garcinia acid because it is the major acidic content in plants belonging to Garcinia species. High percentage of hydroxy citric acid is present in both fruits and leaves, majority in fruit rind (about $23 \%)$. Hydroxy citric acid is isolated from the fruit rind as 
sodium salt and the pure crystalline form of it is produced by using acetone. Hydroxy citric acid contains hydroxyl $(-\mathrm{OH})$ group at $2^{\text {nd }}$ and $3^{\text {rd }}$ carbon atom. It mainly exists in four stereoisomers. Hydroxy citric acid is available commercially, as its calcium salt as the purest form is chemically unstable. ${ }^{9}$ Hydroxy citric acid is also known to increase the serotonin levels in the central nervous system. ${ }^{9,29}$ Considering the fact that hydroxy citric acid, which is a main constituent of Garcinia indica increases serotonin level in rat brain cortex.7,14This information can be attributed to the antidepressant activity demonstrated in both forced swim test and tail suspension test.

\section{CONCLUSIONS}

The study shows that ethanolic extract of Garcinia indica fruit rind possess antidepressant property probably through serotonin increasing activity and the active ingredient which may be contributing to antidepressant action is hydroxy citric acid.

Data sharing statement provided by the authors is available with the full text of this article at jemds.com.

Financial or other competing interests: None.

Disclosure forms provided by the authors are available with the full text of this article at jemds.com.

I would like to acknowledge the teaching and non-teaching staff of my department for their support. I would also like to thank Dr Krishna Kumar, Department of Applied Botany, Mangalore University for carrying out botanical authentication.

\section{REFERENCES}

[1] Pattanayak RD, Sagar R. Depressive disorders in Indian context: a review and clinical update for physicians. J Assoc Physicians India 2014;62(9):55-60.

[2] Gururaj G, Varghese M, Benegal V, et al. National mental health survey of India, 2015-16: summary. Bengaluru, National Institute of Mental Health and Neuro Sciences, NIMHANS Publication 2016.

[3] Wessling A, Ramsberg J. The review of antidepressants. Dental and Pharmaceutical benefits Agtency. Solna:TLV 2008.

[4] Pal SK, Shukla Y. Herbal medicine: current status and the future. Asian Pac J Cancer Prev 2003;4(4):281-8.

[5] Sarris J. Herbal medicines in the treatment of psychiatric disorders: a systematic review. Phytother Res 2007;21(8):703-16.

[6] De Sousa A. Herbal medicines and anxiety disorders: an overview. J Med Plants Stud 2013;1(6):18-23.

[7] Patel M, Antala B, Barua C, et al. Anxiolytic activity of aqueous extract of Garcinia indica in mice. Int J Green Pharm 2013;7(4):332-5.

[8] Milind P, Isha D. Golden benefits of drinking kokam-cola. Int Res J Pharm 2013;4(5):5-9.

[9] Ramachandran HD. Plant profile, phytochemistry and pharmacology of Garcinia indica: a review. Int J Pharm SciRev Res 2014;27(2):376-81.

[10] Jagtap P, Bhise K, Prakya V. A phytopharmacological review on Garcinia indica. J Herb Med 2015;3(4):2-7.
[11] Manikanta P. Methods for isolation of bioactive constituents from Garcinia indica choisy and its medicinal importance: a review. International Journal of Universal Pharmacy and Biosciences 2014;3(1):206-15.

[12] Parasharami VA, Kunder G, Desai N. Recent pharmacological advances of endangered species of South India: Garcinia indica choisy. Journal of Scientific Research \& Reports 2015;8(5):1-10.

[13] Swami SB, Thakor NJ, Patil SC. Kokum (Garcinia indica) and its many functional components as related to the human health: a review. J Food ResTechnol 2014;2(4):130-42.

[14] Ohia SE, Opere CA, LeDay AM, et al. Safety and mechanism of appetite suppression by a novel hydroxycitric acid extract (HCA - SX). Mol Cell Biochem 2002;238(1-2):89-103.

[15] Bhagyashree A, Nayak RP. An In vivo study analyzing the anxiolytic activity of Garcinia indica fruit rind in preclinical models. Indian J Pharm Pharmacol 2020;7(2):95-9.

[16] Khatib NA, Kiran P, Patil PA. Evaluation of anti inflammatory activity of Garcinia indica fruit rind extracts in wistar rats. Int. J Res Ayur Pharm 2010;1(2):449-54.

[17] Deore AB, Sapakal VD, Dashputre NL, et al. Antiulcer activity of Garcinia indica linn fruit rinds. Journal of Applied Pharmaceutical Science 2011;1(5):151-4.

[18] Das A, Hazarika I, Jaikumar S. Antidepressant activity of a polyherbal formulation $\mathrm{r} 013$, in experimental animal model. Int J Phytopharmacol 2014;5(4):267-71.

[19] Swati M, Monalisa J, Abhisek P.Evaluation of antidepressant activity of Eclipta alba using animal models. Asian J Pharm Clin Res 2013;6(Suppl 3):118-20.

[20] Krishnan V,Nestler EJ. The molecular neurobiology of depression. Nature 2008;455(7215):894-902.

[21] Tasman A, Kay J, Lieberman JA, et al. Psychiatry (vol 1). $4^{\text {th }}$ edn. United Kingdom: Wiley Blackwell 2015:917-31.

[22] Brigitta B. Pathophysiology of depression and mechanisms of treatment. Dialogues Clin Neurisci 2002;4(1):7-20.

[23] Cowen PJ, Browning M. What has serotonin to do with depression? World Psychiatry 2015;14(2):158-60.

[24] Tripathi KD. Essentials of medical pharmacology. $7^{\text {th }}$ edn. New Delhi: Brothers Medical Publishers (P) Ltd 2013:455-63.

[25] Vogel HG. Drug discovery and evaluation: Pharmacological assays. Vol. 1. $3^{\text {rd }}$ edn. NewYork: Springer 2008:774-813.

[26] Petit-Demouliere B, Chenu F, Bourin M. Forced swimming test in mice: a review of antidepressant activity. Psychopharmacology 2005;177(3):245-55.

[27] Bogdanova OV, Kanekar S, D'Anci KE, et al. Factors influencing behavior in the forced swim test. Physiol Behav 2013;118:227-39.

[28] Powell TR, Fernandes C, Schalkwyk LC. Depressionrelated behavioral tests. Curr Protoc Mouse Biol 2012;2(2):119-27.

[29] Chuah LO, Ho WY, Beh BK, et al. Updates on antiobesity effect ofGarcinia origin (-)- HCA. Evid Based Complement Alternat Med 2013;2013:751658. 